PROCEEDINGS OF THE

AMERICAN MATHEMATICAL SOCIETY

Volume 125, Number 1, January 1997, Pages 219-228

S 0002-9939(97)03568-5

\title{
A CANTOR-LEBESGUE THEOREM WITH VARIABLE "COEFFICIENTS"
}

\author{
J. MARSHALL ASH, GANG WANG, AND DAVID WEINBERG
}

(Communicated by Christopher D. Sogge)

\begin{abstract}
If $\left\{\phi_{n}\right\}$ is a lacunary sequence of integers, and if for each $n, c_{n}(x)$ and $c_{-n}(x)$ are trigonometric polynomials of degree $n$, then $\left\{c_{n}(x)\right\}$ must tend to zero for almost every $x$ whenever $\left\{c_{n}(x) e^{i \phi_{n} x}+c_{-n}(-x) e^{-i \phi_{n} x}\right\}$ does. We conjecture that a similar result ought to hold even when the sequence $\left\{\phi_{n}\right\}$ has much slower growth. However, there is a sequence of integers $\left\{n_{j}\right\}$ and trigonometric polynomials $\left\{P_{j}\right\}$ such that $\left\{e^{i n_{j} x}-P_{j}(x)\right\}$ tends to zero everywhere, even though the degree of $P_{j}$ does not exceed $n_{j}-j$ for each $j$. The sequence of trigonometric polynomials $\left\{\sqrt{n} \sin ^{2 n} \frac{x}{2}\right\}$ tends to zero for almost every $x$, although explicit formulas are developed to show that the sequence of corresponding conjugate functions does not. Among trigonometric polynomials of degree $n$ with largest Fourier coefficient equal to 1, the smallest one "at" $x=0$ is $4^{n}\left(\begin{array}{c}2 n \\ n\end{array}\right){ }^{-1} \sin ^{2 n}\left(\frac{x}{2}\right)$, while the smallest one "near" $x=0$ is unknown.
\end{abstract}

\section{INTRODUCTION}

The Cantor-Lebesgue theorem asserts that if $\left\{c_{n}\right\}_{n=-\infty}^{\infty}$ is a sequence of complex numbers satisfying the condition that

$$
\lim _{n \rightarrow \infty} c_{n} e^{i n x}+c_{-n} e^{-i n x}=0 \text { for almost all } x,
$$

then $\lim _{n \rightarrow \infty} c_{n}=\lim _{n \rightarrow-\infty} c_{n}=0$. (Actually, Lebesgue obtained the conclusion from a lighter hypothesis. See reference $[A K R]$ for other sufficient hypotheses.)

Here is a reformulation which is easily seen to be equivalent. There does not exist a subsequence of the positive integers $\left\{n_{k}\right\}$ and a sequence of complex numbers $\left\{d_{k}\right\}$ such that

$$
\lim _{k \rightarrow \infty} e^{i 2 n_{k} x}-d_{k}=0 \text { for almost all } x .
$$

This says that in some sense the rapidly oscillating waves $e^{i 2 n_{k} x}$ cannot be well approximated by constants.

Received by the editors July 27, 1995.

1991 Mathematics Subject Classification. Primary 42A05; Secondary 42A50, 42A55.

Key words and phrases. Cantor Lebesgue theorem, conjugate trigonometric series, lacunary trigonometric series, Plessner's theorem, trigonometric polynomials.

The research of J. M. Ash and G. Wang was partially supported by a grant from the Faculty and Development Program of the College of Liberal Arts and Sciences, DePaul University.

(C)1997 American Mathematical Society 
Now let us assume that

$$
\lim _{n \rightarrow \infty} c_{n}(x) e^{i \phi_{n} x}+c_{-n}(-x) e^{-i \phi_{n} x}=0 \text { for almost all } x .
$$

Our goal is to find conditions on $\left\{c_{n}(x)\right\},\left\{c_{-n}(x)\right\}$, and $\left\{\phi_{n}\right\}$ that will force the conclusion that

$$
\lim _{n \rightarrow \infty} c_{n}(x)=\lim _{n \rightarrow \infty} c_{-n}(x)=0 \text { for almost all } x .
$$

We begin with two examples for which condition (3) holds while condition (4) does not:

$$
\begin{aligned}
& \left(\left\{c_{n}(x)\right\},\left\{c_{-n}(-x)\right\},\left\{\phi_{n}\right\}\right)=\left(\{1\},\left\{-e^{-2 i 2^{n} x}\right\},\left\{2^{n}\right\}\right) \text { and } \\
& \left(\left\{c_{n}(x)\right\},\left\{c_{-n}(-x)\right\},\left\{\phi_{n}\right\}\right)=\left(\{n\},\left\{-n e^{-2 i 2^{n} x}\right\},\left\{2^{n}\right\}\right) .
\end{aligned}
$$

These examples show that some restrictions are necessary. Looking at the formulation given by condition (2) above makes it natural to demand that the "coefficients" $\left\{c_{n}(x)\right\}$ and $\left\{c_{-n}(x)\right\}$ oscillate more slowly than the "frequencies" $\left\{\phi_{n}\right\}$. Such a condition would at least eliminate these two examples. However, the problem is a lot more delicate, as the following example shows.

Example 1.1. A sequence of pure sinusoidal waves can be everywhere approximated by a corresponding sequence of lower (nonnegative) frequency trigonometric polynomials. I. e., there is an increasing sequence of integers $\left\{n_{j}\right\}$ and trigonometric polynomials $\left\{P_{j}(x)\right\}$ with $\operatorname{deg} P_{j} \leq n_{j}-j$ such that for every real $x, \lim _{j \rightarrow \infty} e^{i n_{j} x}-P_{j}(x)=0$.

We thank Jean-Pierre Kahane and Charles Fefferman for the following proof.

Proof. Identify the torus with the edge of the unit circle in the complex plane via the mapping $x \rightarrow z=e^{i x}$. For each positive integer $j$, decompose the torus into $F_{j}=\left\{e^{i x}: 0<x<1 / j\right\}$ and its complement $K_{j}$. The function $z^{-j}$ is continuous on $K_{j}$, the set $K_{j}$ is compact and has no interior, and the complement of $K_{j}=\{z:|z| \neq 1\} \cup F_{j}$ is connected, so by a theorem of Mergelyan ([R, page $390])$, there is a polynomial $Q_{j}(x)=\sum_{k=0}^{n_{j}-j} c_{j k} e^{i k x}$ satisfying

$$
\sup _{x \in K_{j}}\left|z^{-j}-Q_{j}(x)\right|<\frac{1}{j} .
$$

Taking complex conjugates and then multiplying by $z^{n_{j}-j}$ gives

$$
\sup _{x \in K_{j}}\left|e^{i n_{j} x}-\sum_{k=0}^{n_{j}-j} c_{j k} e^{i\left(n_{j}-j-k\right) x}\right|<\frac{1}{j} .
$$

Since $\bigcup_{j=1}^{\infty} K_{j}=\lim _{j \rightarrow \infty} K_{j}$ is the entire torus, it remains only to set

$$
P_{j}(x)=\sum_{k=0}^{n_{j}-j} c_{j k} e^{i\left(n_{j}-j-k\right) x} \quad \text { for each } j .
$$

Remark 1.1. Perhaps constructing $Q_{j}$ explicitly will show that $n_{j}$ does not have to increase very rapidly. If $n_{j}$ increases slowly enough, some instances of Conjecture 1.2 below will be false. 
In the other direction, we have the following positive result.

Theorem 1.1. If $\left\{c_{n}(x)\right\}$ and $\left\{c_{-n}(x)\right\}$ are trigonometric polynomials of degree $n$ and if

$$
\lim _{n \rightarrow \infty} c_{n}(x) e^{i \phi_{n} x}+c_{-n}(-x) e^{-i \phi_{n} x}=0 \text { for almost all } x
$$

where the sequence of integers $\left\{\phi_{n}\right\}$ is lacunary $\left(\frac{\phi_{n+1}}{\phi_{n}} \geq \gamma>1\right)$, then

$$
\lim _{n \rightarrow \infty} c_{n}(x)=\lim _{n \rightarrow \infty} c_{-n}(x)=0 \text { for almost all } x .
$$

This motivates the following conjecture.

Conjecture 1.2. If $\left\{c_{n}(x)\right\}$ and $\left\{c_{-n}(x)\right\}$ are trigonometric polynomials of degree $n$ and if

$$
\lim _{n \rightarrow \infty} c_{n}(x) e^{i \phi_{n} x}+c_{-n}(-x) e^{-i \phi_{n} x}=0 \text { for almost all } x,
$$

where the integers $\left\{\phi_{n}\right\}$ satisfy $\liminf _{n \rightarrow \infty} \frac{\phi_{n}}{n} \geq g>1$, then

$$
\lim _{n \rightarrow \infty} c_{n}(x)=\lim _{n \rightarrow \infty} c_{-n}(x)=0 \text { for almost all } x .
$$

The proof of the theorem is entertaining, but sheds no light on the much more delicate conjecture.

To further motivate the theorem and the conjecture, and in particular, the choice of the minus sign in the argument of $c_{-n}$ we will now summarize some results obtained elsewhere [AWa].

Sequences like those appearing in the hypothesis of the Cantor-Lebesgue theorem appear naturally when considering differences of successive partial sums of a trigonometric series, while sequences like those appearing in the hypotheses of the theorem and the conjecture above appear naturally when considering similar differences for subsequences of partial sums. We will make this explicit. Let $\left\{A_{n}(x)\right\}:=\left\{c_{n} e^{i n x}+c_{-n} e^{-i n x}\right\}$. An immediate corollary of the Cantor-Lebesgue theorem is the fact that if the sequence of partial sums $\left\{s_{n}(x)\right\}$, where $s_{n}(x)=$ $\sum_{v=1}^{n} A_{v}(x)$, of the trigonometric series

$$
c_{o}+\sum_{n=1}^{\infty} A_{n}(x)
$$

converges almost everywhere, then $\left\{\left|c_{n}\right|+\left|c_{-n}\right|\right\}$ tends to 0 as $n \rightarrow \infty$. Suppose instead that only the subsequence of partial sums $\left\{s_{n^{2}-1}(x)\right\}$ converges almost everywhere. Theorem 10 of [AWa] essentially says that

if the subsequence $\left\{s_{n^{2}-1}\right\}$ of a trigonometric series of power series type converges almost everywhere, then the coefficients must satisfy $\limsup \left|c_{n}\right|^{\frac{1}{\sqrt{n}}} \leq 1$.

$$
|n| \rightarrow \infty
$$

However, from the a.e. convergence of $\left\{s_{n^{2}-1}(x)\right\}$ it follows that

$$
s_{(n+1)^{2}-1}(x)-s_{n^{2}-1}(x)=\left(\sum_{v=0}^{2 n} c_{v+n^{2}} e^{i v x}\right) e^{i n^{2} x}+\left(\sum_{v=0}^{2 n} c_{-v-n^{2}} e^{-i v x}\right) e^{-i n^{2} x}
$$


tends to zero almost everywhere. If the conjecture holds with $\phi_{2 n}=n^{2}$, it will then follow that

$$
\lim _{n \rightarrow \infty} \sum_{v=0}^{2 n} c_{v+n^{2}} e^{i v x}=\lim _{n \rightarrow \infty} \sum_{v=0}^{2 n} c_{-v-n^{2}} e^{-i v x}=0 \text { for almost every } x .
$$

It easily follows from this and the proof of Theorem 10 that statement (6) remains valid when the underlined words are omitted. The central question of this paper arose from the authors' efforts to strengthen Theorem 10 in a similar way.

In sections 3 and 4 we study the very interesting sequence of functions

$$
\left\{\sqrt{n} \sin ^{2 n} \frac{x}{2}\right\} \text {. }
$$

In section 3 we find this neat formula for the conjugate functions:

$$
\left(\sqrt{n} \sin ^{2 n} \frac{x}{2}\right)^{\sim}=2 n^{3 / 2} 4^{-n}\left(\begin{array}{c}
2 n \\
n
\end{array}\right) \sin ^{2 n}\left(\frac{x}{2}\right) \int_{x / 2}^{\pi / 2} \frac{1}{\sin ^{2 n} t} d t .
$$

This formula makes it very easy to show that the conjugates do not converge to zero at any point of $(-\pi, 0) \cup(0, \pi)$, even though the original sequence of functions converges to zero on $(-\pi, \pi)$. The explanation for this strange behavior is that when the $n$th term of the sequence (7) is written as a trigonometric polynomial of degree $n$, the constant term, $\sqrt{n} 4^{-n}\left(\begin{array}{c}2 n \\ n\end{array}\right)$, is almost $1 / \sqrt{\pi}$, while for each $x \in(-\pi, \pi)$, the sequence (7) tends rapidly to zero. In other words, the sequence (7) is of more or less constant size when viewed from a trigonometric series point of view, but is "unreasonably small" when viewed from the point of view of pointwise convergence.

In section 4 we continue the study of the sequence (7), by contrasting two seemingly quite similar situations. In both, trigonometric polynomials of degree $n$ with largest Fourier coefficient having modulus equal to 1 are considered. If we look for the smallest such function "at" $x=0$, then $4^{n}\left(\begin{array}{c}2 n \\ n\end{array}\right)^{-1} \sin ^{2 n}\left(\frac{x}{2}\right)$ is extremal, but if we look for the smallest such function "near" $x=0$, it is not.

In section 5 we suggest a line of research inspired by the results of sections 3 and 4 which might lead to a counterexample to certain cases of the main conjecture given above.

\section{Proof of Theorem 1.1}

Let the sequence of integers $\left\{\phi_{n}\right\}$ satisfy $\phi_{1}>0, \frac{\phi_{n+1}}{\phi_{n}} \geq \gamma_{0}>1$ and suppose that

$$
\lim _{n \rightarrow \infty} A_{n}(x)=0 \text { for almost all } x,
$$

where $A_{n}(x)=c_{n}(x) e^{i \phi_{n} x}+c_{-n}(-x) e^{-i \phi_{n} x}$ with $c_{n}(x)$ and $c_{-n}(x)$ being trigonometric polynomials with complex coefficients of degree $n$. Our goal is to show that

$$
\lim _{n \rightarrow \infty} c_{n}(x)=0 \text { for almost all } x .
$$

(Notice that $c_{-n}(-x)=e^{i \phi_{n} x}\left(A_{n}(x)-c_{n}(x) e^{i \phi_{n} x}\right)$, so that from (8), (9), and $\left|e^{i \phi_{n} x}\right|=1$, it is immediate that $\lim _{n \rightarrow \infty} c_{-n}(x)=0$ a.e. also.)

The conjugate of $e^{i n x}$ is $\widetilde{e^{i n x}}=(-i \operatorname{sgn} n) e^{i n x}$ so that

$$
A_{n}(x)+i \tilde{A}_{n}(x)=2 c_{n}(x) e^{i \phi_{n} x} .
$$

This identity forms the link between statements (8) and (9). Thus, for example, we would have an immediate proof if it were true that whenever $\lim _{n \rightarrow \infty} A_{n}(x)=0$ 
a.e. for trigonometric polynomials $A_{n}$ with $\operatorname{deg} A_{n}=n$, then $\lim _{n \rightarrow \infty} \tilde{A}_{n}(x)=0$ a.e. Unfortunately, this is false. (Let $\left\{A_{n}\right\}$ approximate the Dirac measure $\delta$ in such a way that $\tilde{A}_{n} \rightarrow \tilde{\delta}$. For another example, see the next section below.) So the following more circuitous argument is needed.

1. From the hypothesis (8) it follows that $S(x)=\sum \frac{A_{n}(x)}{n^{2}}$ converges a.e.

2. By a theorem of Plessner ([Z, page 216]), it follows that $\tilde{S}(x)=\sum \frac{\tilde{A}_{n}(x)}{n^{2}}$ also converges a.e.

3. Hence $\tilde{A}_{n}(x) n^{-2} \rightarrow 0$ a.e.

4. It follows from 3. and Egoroff's theorem that for each $\lambda \in(0,1)$ there is a constant $B=B(\lambda)$ such that for all $n,\left|\tilde{A}_{n}(x) n^{-2}\right| \leq B$ for all $x$ in some subset of $(-\pi, \pi]$ of measure at least $2 \pi \lambda$.

5. It also follows from the hypothesis and Egoroff's theorem that there is a constant $B^{\prime}=B^{\prime}(\lambda)$ such that for all $n,\left|A_{n}(x)\right| \leq\left|A_{n}(x) n^{-2}\right| \leq B^{\prime}$ for all $x$ in some subset of $(-\pi, \pi]$ of measure at least $2 \pi \lambda$; so by identity (10) we find that there is a constant $B^{\prime \prime}=B^{\prime \prime}(\lambda)$ such that for all $n,\left|c_{n}(x)\right| \leq B^{\prime \prime} n^{2}$ for all $x$ in some subset of $(-\pi, \pi]$ of measure at least $2 \pi \lambda$.

6. Pick $\gamma_{1} \in\left(1, \gamma_{0}\right)$. By 5. and a theorem of Paul Cohen, there is a constant $b$ such that for every $n$ and every $x,\left|c_{n}(x)\right| \leq b n^{2}\left(\gamma_{1}\right)^{n}$ ([AWa, Lemma 9], and [AWe, pages 404-406]). Similarly, for every $n$ and every $x,\left|c_{-n}(-x)\right| \leq b n^{2}\left(\gamma_{1}\right)^{n}$.

7. Denoting differentiation with respect to $x$ by a prime and applying a theorem of Bernstein, we have for every $n$ and every $x,\left|c_{n}^{\prime}(x)\right| \leq b n^{3}\left(\gamma_{1}\right)^{n}$ ([Z, page 11]). By picking $\gamma \in\left(\gamma_{1}, \gamma_{0}\right)$ and a constant $c$ appropriately, we may present this conclusion more neatly by saying that we have

$$
\text { for every } n \text { and every } x,\left|c_{n}^{\prime}(x)\right| \leq c \gamma^{n} \text {. }
$$

Similarly,

$$
\text { for every } n \text { and every } x,\left|c_{-n}^{\prime}(x)\right| \leq c \gamma^{n} \text {. }
$$

Roughly speaking, the "coefficients" $c_{n}$ and $c_{-n}$ are just about constant within a period of the fast oscillators $e^{i \phi_{n} x}$ and $e^{-i \phi_{n} x}$ (an interval of length $\frac{2 \pi}{\phi_{n}}$ ), so that at most points of each period, $A_{n}$ is small by virtue of its two summands each being small rather than by virtue of the cancellation of one with the other. It remains only to make this argument rigorous.

8. Assume that the conclusion is false, so that $\limsup _{n \rightarrow \infty}\left|c_{n}(x)\right|>0$ on $F$, $|F|>0$. The set of points $x$ where $\lim _{n \rightarrow \infty} A_{n}(x)=0$ has full measure; so by Egoroff's theorem, a set $E$ of measure exceeding the measure of the complement of $F$ can be picked so that $A_{n}(x)$ converges to zero uniformly on $E$. Since almost every point of $E$ is a point of density of $E$, there is such a point $x$ in $E \cap F$. Since $x \in F$,

$$
\limsup _{n \rightarrow \infty}\left|c_{n}(x)\right|>0
$$

However, since $x$ is a point of density of $E$, for each sufficiently large $k$ we may pick $y_{k}$ also in $E$ so that

$$
y_{k}-x \in\left[\frac{\pi}{4 \phi_{k}}, \frac{\pi}{2 \phi_{k}}\right]
$$


Now, following a pretty remark of Roger Cooke $[\mathrm{C}]$, we treat the equations defining $A_{k}$ at $x$ and $y_{k}$ as a system of 2 linear equations in the 2 unknowns $c_{k}(x)$ and $c_{-k}(-x)$ :

$$
\left\{\begin{array}{ccc}
c_{k}(x) e^{i \phi_{k} x}+c_{-k}(-x) e^{-i \phi_{k} x} & = & A_{k}(x) \\
c_{k}(x) e^{i \phi_{k} y_{k}}+c_{-k}(-x) e^{-i \phi_{k} y_{k}} & = & A_{k}\left(y_{k}\right)+\Delta
\end{array}\right.
$$

where $\Delta=\left(c_{k}(x)-c_{k}\left(y_{k}\right)\right) e^{i \phi_{k} y_{k}}+\left(c_{-k}(-x)-c_{-k}\left(-y_{k}\right)\right) e^{-i \phi_{k} y_{k}}$. Solving for $c_{k}(x)$ yields

$$
c_{k}(x)=\frac{e^{-i \phi_{k} y_{k}}\left(A_{k}\left(y_{k}\right)+\Delta\right)-e^{-i \phi_{k} x} A_{k}(x)}{2 i \sin \phi_{k}\left(x-y_{k}\right)} .
$$

Take absolute values. The denominator exceeds $2 \sin \pi / 4$ because of relation (14). Use the triangle inequality, the mean value theorem, and relations (11), (12), and (14) to estimate $\Delta$. We conclude that there must be a constant $C$ such that $\left|c_{k}(x)\right| \leq$ $C\left\{\left|A_{k}\left(y_{k}\right)\right|+M_{k} \frac{\gamma^{k}}{\phi_{k}}+\left|A_{k}(x)\right|\right\}$. Since all three terms inside the curly brackets tend to 0 as $k$ increases, this contradicts relation (13).

\section{Small Functions With Large CONJUGates}

Theorem 3.1. For each $n=1,2, \ldots$, let $f_{n}(x)=k_{n} \sin ^{2 n}\left(\frac{x}{2}\right)$, where $k_{n}=$ $\frac{2 n-1}{n} 4^{n}\left(\begin{array}{c}2 n \\ n\end{array}\right)^{-1}$. The sequence of functions $\left\{f_{n}(x)\right\}$ converges to zero for each $x \in$ $(-\pi, \pi)$ while the sequence of functions $\left\{\tilde{f}_{n}(x)\right\}$ converges to zero for no $x$ in $(-\pi, 0) \cup(0, \pi)$.

Lemma 3.2. The conjugate of $f_{n}(x)=k_{n} \sin ^{2 n}\left(\frac{x}{2}\right)$ is

$$
\tilde{f}_{n}(x)=-2(2 n-1) \sin ^{2 n}\left(\frac{x}{2}\right) \int_{x / 2}^{\pi / 2} \frac{d t}{\sin ^{2 n} t} \text {. }
$$

Proof of Theorem 3.1. By Stirling's formula, $k_{n} \simeq 2 \sqrt{\pi n}$ as $n \rightarrow \infty$, whence it is clear that $\left\{f_{n}(x)\right\}$ converges to zero for each $x \in(-\pi, \pi)$.

Let $x \in(0, \pi)$. Set $t=\frac{\theta}{2}$, so that we may use a well known reduction formula to find that

$$
\int_{x / 2}^{\pi / 2} \frac{d t}{\sin ^{2 n} t}=\frac{1}{2 n-1} \frac{\cos \frac{x}{2}}{\sin ^{2 n-1} \frac{x}{2}}+\frac{2 n-2}{2 n-1} \int_{x / 2}^{\pi / 2} \frac{d t}{\sin ^{2 n-2} t} \geq \frac{1}{2 n-1} \frac{\cos \frac{x}{2}}{\sin ^{2 n-1} \frac{x}{2}} .
$$

By the lemma we have

$$
\tilde{f}_{n}(x) \leq-2(2 n-1) \sin ^{2 n}\left(\frac{x}{2}\right)\left(\frac{1}{2 n-1} \frac{\cos \frac{x}{2}}{\sin ^{2 n-1} \frac{x}{2}}\right)=-2 \sin \frac{x}{2} \cos \frac{x}{2}=-\sin x,
$$

so that $\lim _{n \rightarrow \infty} \tilde{f}_{n}(x) \neq 0$ when $x \in(0, \pi)$. Since each $\tilde{f}_{n}(x)$ is odd, we also see that $\lim _{n \rightarrow \infty} \tilde{f}_{n}(x) \neq 0$ when $x \in(-\pi, 0)$. 
Proof of Lemma 3.2. From $\sin \left(\frac{x}{2}\right)=\frac{e^{i x / 2}-e^{-i x / 2}}{2 i}$ it follows that $f(x)=\sin ^{2 n}\left(\frac{x}{2}\right)=$ $4^{-n}\left(\begin{array}{c}2 n \\ n\end{array}\right)+2 \cdot 4^{-n} \sum_{m=1}^{n}\left(\begin{array}{c}2 n \\ n-m\end{array}\right)(-1)^{m} \cos m x$, so that

$$
\tilde{f}(x)=2 \cdot 4^{-n} \sum_{m=1}^{n}\left(\begin{array}{c}
2 n \\
n-m
\end{array}\right)(-1)^{m} \sin m x .
$$

Let $S(x)=f(x)+i \tilde{f}(x)$ and let $z=e^{i x}$. Then $S$ may be thought of as a function of $z$ so that the substitution $k=n-m$ yields

$$
S(z)=4^{-n}\left(\begin{array}{c}
2 n \\
n
\end{array}\right)+2 \cdot 4^{-n}(-z)^{n} g\left(\frac{-1}{z}\right),
$$

where $g(z)=\sum_{k=0}^{n-1}\left(\begin{array}{c}2 n \\ k\end{array}\right) z^{k}$. The whole proof turns on the lucky fact that $g$ satisfies an easily solved first order differential equation. Direct calculation shows that

$$
(1+z) g^{\prime}(z)-2 n g(z)=-\left(\begin{array}{c}
2 n \\
n
\end{array}\right) n z^{n-1}
$$

Solving this equation by first solving the associated homogeneous differential equation and then applying the method of variation of parameters leads to

$$
g(z)=(1+z)^{2 n}\left(1-\left(\begin{array}{c}
2 n \\
n
\end{array}\right) n \int_{0}^{z} t^{n-1}(1+t)^{-2 n-1} d t\right) .
$$

Since $z=e^{i x}$,

$$
g\left(\frac{-1}{z}\right)=g\left(e^{i(\pi-x)}\right)=\left(1-e^{-i x}\right)^{2 n}\left(1-\left(\begin{array}{c}
2 n \\
n
\end{array}\right) n h(x)\right),
$$

where $h(x)=\int_{0}^{e^{i(\pi-x)}} t^{n-1}(1+t)^{-2 n-1} d t$. Since the integrand is analytic when $t \neq-1$, when $x \neq 0$, we may choose our path of integration to be the line segment from 0 to 1 followed by the arc of the unit circle from 1 to $e^{i(\pi-x)}$. This gives

$$
\begin{gathered}
h(x)=\int_{0}^{1} t^{n-1}(1+t)^{-2 n-1} d t+\int_{0}^{\pi-x} \frac{e^{i(n-1) \theta}}{\left(1+e^{i \theta}\right)^{2 n+1}} i e^{i \theta} d \theta \\
=(2 n)^{-1}\left\{\left(\begin{array}{c}
2 n \\
n
\end{array}\right)^{-1}+4^{-n}\right\}+\frac{i}{2} 4^{-n} \int_{0}^{\pi-x} \frac{e^{-i \frac{\theta}{2}}}{\cos ^{2 n+1}\left(\frac{\theta}{2}\right)} d \theta \\
=(2 n)^{-1}\left\{\left(\begin{array}{c}
2 n \\
n
\end{array}\right)^{-1}+4^{-n} \frac{1}{\sin ^{2 n}\left(\frac{x}{2}\right)}\right\}+\frac{i}{2} 4^{-n} \int_{0}^{\pi-x} \frac{1}{\cos ^{2 n}\left(\frac{\theta}{2}\right)} d \theta .
\end{gathered}
$$

Substitute this into equation (16) and note that $\left(1-e^{-i x}\right)^{2 n}=\sin ^{2 n}\left(\frac{x}{2}\right)(-1 / z)^{n} 4^{n}$ to get

$g\left(\frac{-1}{z}\right)=\sin ^{2 n}\left(\frac{x}{2}\right)(-1 / z)^{n} 4^{n}\left[\frac{1}{2}-\frac{1}{2} \frac{\left(\begin{array}{c}2 n \\ n\end{array}\right)}{4^{n}} \frac{1}{\sin ^{2 n}\left(\frac{x}{2}\right)}-\frac{i}{2} \frac{n\left(\begin{array}{c}2 n \\ n\end{array}\right)}{4^{n}} \int_{0}^{\pi-x} \frac{1}{\cos ^{2 n}\left(\frac{\theta}{2}\right)} d \theta\right]$.

Finally, substitute this into equation (15) to get

$$
S(z)=\sin ^{2 n}\left(\frac{x}{2}\right)-i 4^{-n}\left(\begin{array}{c}
2 n \\
n
\end{array}\right) n \sin ^{2 n}\left(\frac{x}{2}\right) \int_{0}^{\pi-x} \frac{1}{\cos ^{2 n}\left(\frac{\theta}{2}\right)} d \theta
$$

Multiply through by $k_{n}$ and make the substitution $t=\frac{\pi-\theta}{2}$ to establish the lemma. 


\section{The Remarkable functions $\sin ^{2 n}\left(\frac{x}{2}\right)$}

Definition 4.1. Say that a function $f(x)$ is asymptotic to 0 of order $n$ at $a$ if $n$ is the smallest real number such that $\lim _{\sup _{x \rightarrow a}} \frac{|f(x)|}{|x-a|^{n}}$ is finite.

Lemma 4.1. The only trigonometric polynomials of degree $n$,

$$
c_{0}+\sum_{k=1}^{n} a_{k} \cos k x+b_{k} \sin k x
$$

where

$$
\max \left\{\left|c_{0}\right|,\left|a_{1}\right|, \cdots,\left|a_{n}\right|,\left|b_{1}\right|, \cdots,\left|b_{n}\right|\right\}=1,
$$

which are asymptotic to 0 of order $2 n$ at 0 are

$$
P_{\alpha}(x):=e^{i \alpha} \frac{2^{n}}{\left(\begin{array}{c}
2 n \\
n
\end{array}\right)}(1-\cos x)^{n} .
$$

In particular, if we normalize by demanding that $c_{0}$ be real and nonnegative, then the unique trigonometric polynomial of degree $n$ satisfying (17) and being asymptotic to 0 of order $2 n$ at 0 is

$$
P_{0}(x):=\frac{2^{n}}{\left(\begin{array}{c}
2 n \\
n
\end{array}\right)}(1-\cos x)^{n},
$$

which has constant term 1.

Proof. Replacing the trigonometric functions sin and cos by their Taylor expansions, we see that the degree $n$ trigonometric polynomial $P$ can be written as

$$
\begin{aligned}
c_{0}+\sum_{k=1}^{n} \sum_{m=0}^{n}\left(a_{k}(-1)^{m} \frac{(k x)^{2 m}}{(2 m) !}+b_{k}(-1)^{m} \frac{(k x)^{2 m+1}}{(2 m+1) !}\right)+O\left(x^{2 n+2}\right) \\
=c_{0}+\sum_{m=0}^{n}\left((-1)^{m} \frac{x^{2 m}}{(2 m) !}\left\{\sum_{k=1}^{n} a_{k} k^{2 m}\right\}\right. \\
\left.+(-1)^{m} \frac{x^{2 m+1}}{(2 m+1) !}\left\{\sum_{k=1}^{n} b_{k} k^{2 m+1}\right\}\right)+O\left(x^{2 n+2}\right) .
\end{aligned}
$$

Thus for $P$ to be asymptotic to 0 of order $2 n$ at 0 , the $a_{k}$ 's and $b_{k}$ 's must satisfy the following equations:

$$
\begin{aligned}
& \left(\begin{array}{cccc}
1 & 1 & \cdots & 1 \\
1^{2} & 2^{2} & \cdots & n^{2} \\
\cdots & \cdots & \cdots & \cdots \\
1^{2 n-2} & 2^{2 n-2} & \cdots & n^{2 n-2}
\end{array}\right)\left(\begin{array}{c}
a_{1} \\
a_{2} \\
\cdots \\
a_{n}
\end{array}\right)=\left(\begin{array}{c}
-c_{0} \\
0 \\
\cdots \\
0
\end{array}\right), \\
& \left(\begin{array}{cccc}
1 & 2 & \cdots & n \\
1^{3} & 2^{3} & \cdots & n^{3} \\
\cdots & \cdots & \cdots & \cdots \\
1^{2 n-1} & 2^{2 n-1} & \cdots & n^{2 n-1}
\end{array}\right)\left(\begin{array}{c}
b_{1} \\
b_{2} \\
\cdots \\
b_{n}
\end{array}\right)=\left(\begin{array}{c}
0 \\
0 \\
\cdots \\
0
\end{array}\right) .
\end{aligned}
$$

The determinant of the top $n$ by $n$ matrix is a Vandermonde determinant and hence is not zero. Similarly, the determinant of the bottom $n$ by $n$ matrix is also nonzero, since this determinant is a nonzero multiple of a Vandermonde determinant, as can 
be seen by factoring $j$ out of the $j$ th column, $j=2,3, \ldots, n$. Thus all the $b_{k}$ 's must be zero and all the $a_{k}$ 's are uniquely determined once $c_{0}$ is fixed.

Now observe that replacing $\cos x$ by $1-\frac{x^{2}}{2}+O\left(x^{4}\right)$ in $P_{\alpha}$ shows $P_{\alpha}$ to be asymptotic to 0 of order $2 n$ at 0 . Furthermore, replacing $(1-\cos x)^{n}$ by $2^{n}\left(\frac{e^{i x / 2}-e^{-i x / 2}}{2 i}\right)^{2 n}$ and using the binomial expansion show that the constant term of $P_{\alpha}$ is $e^{i \alpha}$, and, since $\left(\begin{array}{c}2 n \\ n\end{array}\right)>\left(\begin{array}{c}2 n \\ j\end{array}\right)$ for $j \in[0,2 n] \backslash\{n\}$, all the other coefficients of $P_{\alpha}$ are of smaller modulus. In short, $P_{\alpha}$ has all the required properties, and the uniqueness of $P_{\alpha}$ is guaranteed by the argument of the preceding paragraph.

Question. Fix any $\varepsilon \in(0, \pi]$ and let $I_{\varepsilon}:=[-\varepsilon, \varepsilon]$. Find all trigonometric polynomials of degree $n$ satisfying condition (17) and having minimum suprema on $I_{\varepsilon}$. Perhaps the most interesting special case of this question occurs when $\varepsilon=\pi$.

Remarks. 1. This question has the flavor of the Heisenberg Uncertainty Principle in the sense that we are looking for a small function with a large Fourier transform. Here we are specifying a kind of supremum norm on both function and transform, but it is clear that many similar questions can be raised. For example, consider trigonometric polynomials of fixed degree and fixed $\ell_{p}$ norm on the transform side, and attempt to find the ones with extremal $L_{q}[-\varepsilon, \varepsilon]$ norm on the function side, where $q$ may be $p$ or the conjugate exponent $p /(p-1)$ or possibly something else.

2. Let

$$
\|P\|_{\varepsilon}:=\sup _{x \in I_{\varepsilon}}|P(x)| .
$$

We were led to this question by thinking about Lemma 4.1 above. It seemed reasonable to conjecture that for each integer $n>0$ there is a small positive number $\varepsilon=\varepsilon(n)$ such that the only trigonometric polynomials of degree $n$ satisfying (17) for which $\|\cdot\|_{\varepsilon}$ is minimal are those of the form $P_{\alpha}$. We thank Professor Peter Borwein for showing us that this is not the case. To see this, fix $\varepsilon \in(0, \pi / 2)$ and $n$. Then $P_{0}(x)$ is 0 at $x=0$, increasing on $[0, \varepsilon]$, even, and of the form $1+$ $a_{1} \cos x+\ldots+a_{n} \cos n x$, where $1>\left|a_{1}\right|>\ldots>\left|a_{n}\right|$. Then if $d>0$ and $x \in I_{\varepsilon}$, $-d \leq P_{0}(x)-d \cos x \leq P_{0}(\varepsilon)-d \cos \varepsilon$, so choosing $d$ small enough produces a new trigonometric polynomial, $P_{0}(x)-d \cos x$, which still satisfies conditions (17) and which satisfies $\left\|P_{0}(x)-d \cos x\right\|_{\varepsilon}<\left\|P_{0}(x)\right\|_{\varepsilon}$. (To be precise, $d$ must be so small that $d<P_{0}(\varepsilon)=\left\|P_{0}(x)\right\|_{\varepsilon}$ and that $\left|a_{1}-d\right|<1$.)

\section{A Counterexample For the main COnjeCture?}

In the previous section, the function $f_{n}(x)=k_{n} \sin ^{2 n}\left(\frac{x}{2}\right)$ was seen to arise naturally as the most asymptotically small at 0 among the real trigonometric polynomials of degree $n$ with constant term $k_{n}\left(\begin{array}{c}2 n \\ n\end{array}\right) 4^{-n}$. (See section 3 above for the definition of $k_{n}$.) To relate this to the main conjecture, define $c_{n}(x)$ and $c_{-n}(x)$ respectively by $2 c_{n}(x)=f_{n}(x)+i \tilde{f}_{n}(x)$ and $2 c_{-n}(-x)=f_{n}(x)-i \tilde{f}_{n}(x)$. Finally set $A_{n}(x)=c_{n}(x) e^{i 0 x}+c_{-n}(-x) e^{-i 0 x}$. Then we can recast the result of section 2 by saying that we have here a counterexample to the main conjecture in the special case when all $\phi_{n}=0$. (Of course, this isn't fair since the condition $\frac{\phi_{n}}{n} \geq g>1$ fails.)

Motivated by this, start with, say, $c_{n}(x) e^{i 2 n x}+c_{-n}(-x) e^{-i 2 n x}$, where the coefficients of the polynomials $c_{n}$ and $c_{-n}$ are to be determined. Next define $a_{n}$ and 
$\tilde{a}_{n}$ from the system

$$
\begin{array}{ll}
2 c_{n}(x) e^{i 2 n x} & =a_{n}(x)+i \tilde{a}_{n}(x), \\
2 c_{-n}(-x) e^{-i 2 n x} & =a_{n}(x)-i \tilde{a}_{n}(x) .
\end{array}
$$

Now $a_{n}(x)$ has the form $\sum_{v=2 n}^{3 n} d_{v} e^{i v x}$. Use the Maclaurin expansion methods of section 3 to make $a_{n}$ real and asymptotically small of order $2 n$ at zero, while having largest coefficient equal to 1 . Then $a_{n}$ will be "unreasonably small", just as $f_{n}$ was. If $\tilde{a}_{n}$ turns out to be "much larger" than $a_{n}$, just as $\tilde{f}_{n}$ was "much larger" than $f_{n}$, then the main conjecture will be false when $\phi_{n}=2 n$. Estimating the size of $\tilde{a}_{n}$ seems to require a lot of technical power. (In particular, the methods used in section 3 don't appear to work here.)

\section{REFERENCES}

[AKR] J. M. Ash, R. P. Kaufman, and E. Rieders, The Cantor-Lebesgue property, Israel J. of Math., 84(1993), 179-191. MR 94m:42007

[AWa] J. M. Ash and G. Wang, One and two dimensional Cantor-Lebesgue type theorems, Trans. Amer. Math. Soc. (to appear). CMP 96:03

[AWe] J. M. Ash and G. V. Welland, Convergence, uniqueness, and summability of multiple trigonometric series, Trans. Amer. Math. Soc. 163(1972), 401-436. MR 45:9057

[C] R. Cooke, The Cantor-Lebesgue theorem, Amer. Math. Monthly 86(1979), 558-565. MR 81b:42019

[R] W. Rudin, Real and Complex Analysis, 3rd ed., McGraw-Hill, New York, 1987. MR 88k:00002

[Z] A. Zygmund, Trigonometric Series, Vol. 2, 2nd rev. ed., Cambridge Univ. Press, New York, 1959. MR 21:6498

(J. M. Ash and G. Wang) Department of Mathematics, DePaul University, Chicago, ILLINOIS 60614-3504

E-mail address: mash@math.depaul.edu

E-mail address: gwang@math.depaul.edu

(D. Weinberg)Department of Mathematics, Texas Tech University, Lubbock, Texas 79409-1042

E-mail address: weinberg@math.ttu.edu 